\title{
A Synchronization Algorithm for TDD Active Optical Distribution Systems
}

\author{
Wei Wang, Zhi-de Li \\ Department of Electronic Engineering, Tsinghua University, Beijing, China \\ E-mail: washingway@163.com, li.zhide@sz.tsinghua.edu.cn
}

\begin{abstract}
As a new wireless network coverage device, the active optical distribution system used for coverage enhancement of GSM, TD-SCDMA and TD-LTE wireless networks has many merits, such as green, micro power, multi-antenna and multipoint uniform depth coverage when compared with the traditional indoor distribution system. As an extension system of the base station wireless network, the active optical distribution system is required to obtain accurate time synchronization information of the TDD system in order to work normally according to the uplink and downlink time slots alignment of the base station. Therefore synchronization problem becomes a primary issue in the active optical distribution system. In this paper, TD-LTE time-frequency synchronization process and primary and secondary synchronous detection algorithm are firstly investigated. Then the time-frequency synchronization algorithm and the influence of frequency offset on the timing synchronization algorithm are analyzed. The simulation results show that a range of frequency offset has little effect on the timing synchronization accuracy. In the TDD-LTE secondary synchronization signal detection process, the traditional method is 168 groups of secondary synchronization sequence and the received sequence to do a length of 62-bit crosscorrelation operation, whose computation is huge. In this paper, a new parity separation-based method is proposed,
\end{abstract}

which is 31 times the length of 31-bit synchronous crosscorrelation operation. The proposed method is proved to have lower complexity i.e., one-tenth calculation times of the traditional method.

Keywords-active optical distribution system; primary synchronization; secondary synchronization

\section{INTRODUCTION}

As a new indoor distributed system solution, the active optical distribution system has the characteristics of small loss and large bandwidth of optical fiber transmission. It has the advantages of supporting multi-standard, multi-service access and multi-network integration, and compatibility and expansibility. At the same time the application of fiber and cable instead of the traditional coaxial cable for indoor distribution construction, can reduce the difficulty of construction, speed up the indoor distribution of construction, and can quickly achieve low-cost settlement of villages, buildings, residential streets, elevators and office buildings and other special areas of $2 \mathrm{G}, 3 \mathrm{G}$ and $4 \mathrm{G}$ signal depth coverage. see Fig. 1.

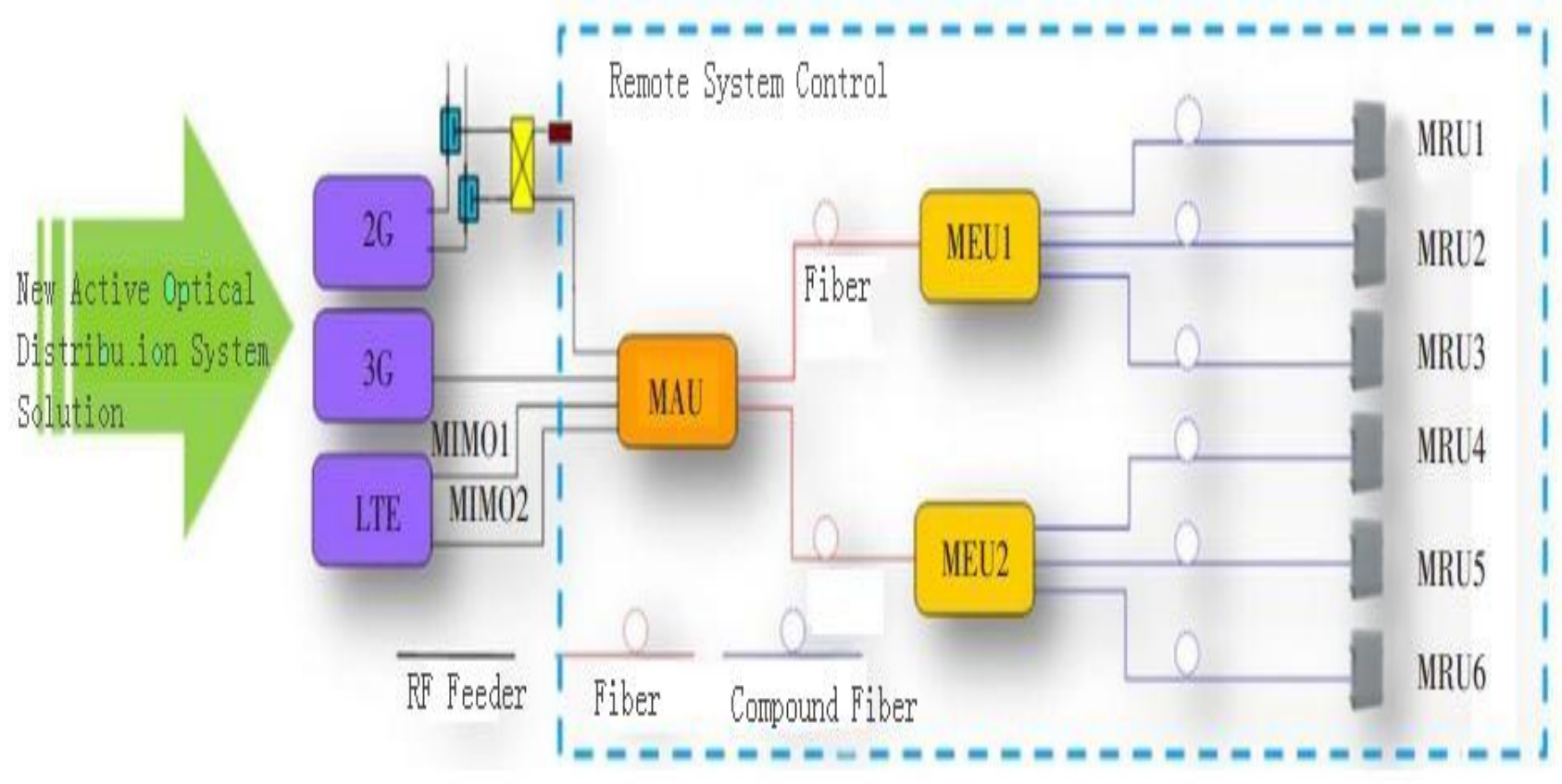

Figure 1. Active optical distribution system to achieve the MIMO of LTE 
Characteristics of the active optical distribution system:

- The construction of optical fiber cable is convenient and concealed, the coordination difficulty of the property is greatly reduced.

- "Low-power, multi-antenna" approach to achieve accurate coverage.

- RF unit on the antenna side, improving the system SNR.

- Network monitoring, improving maintenance and optimization efficiency.

- Multi-service solution.

- Reducing the requirements of passive components.

China Mobile's active optical distribution system involves TD-SCDMA and TD-LTE TDD systems standard, in which synchronous problem is a very critical problem, Only the TDD system get accurate timing synchronization signal, can the distribution system operate normally. Thus the synchronization problem becomes the primary issue in the active optical distribution system. In this paper, we will study the synchronization signal of the TDD system.

\section{TD-LTE FRAME STRUCTURE AND UPLINK AND DOWNLINK TIME SLOT RATIO}

The LTE standard supports two duplex modes: FDD (type 1) and TDD (type 2). In TDD mode, it is a TD-LTE system. The frame structure in TDD mode (type 2) is shown below $^{[1]}$, see Fig. 2 .

The TD-LTE system allocates uplink and downlink resources in units of subframe.3GPP protocol provides seven kinds of uplink and downlink slot matching and nine special subframe configuration in the TD-LTE system.

The seven types of uplink and downlink time slot configurations are shown as below, see Table I.

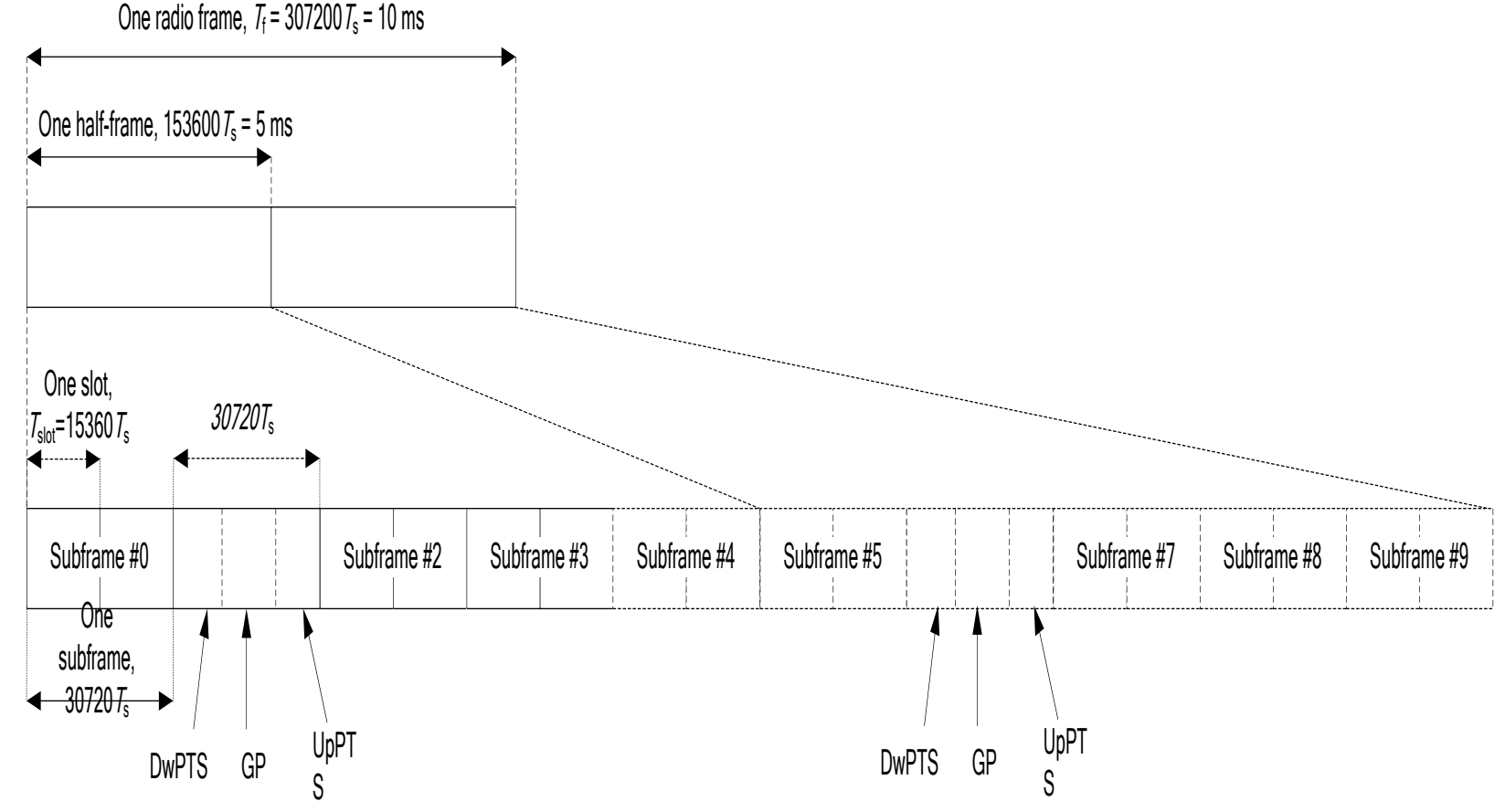

Figure 2. Frame structure type 2 (for $5 \mathrm{~ms}$ switch-point periodicity)

TABLE I. Uplink-downlink configurations

\begin{tabular}{|c|c|c|c|c|c|c|c|c|c|c|c|}
\hline \multirow{2}{*}{$\begin{array}{l}\text { Uplink-downlink } \\
\text { configuration }\end{array}$} & \multirow{2}{*}{$\begin{array}{l}\text { Downlink-to- } \\
\text { Uplink } \\
\text { Switch-point } \\
\text { periodicity }\end{array}$} & \multicolumn{10}{|c|}{ Subframe number } \\
\hline & & 0 & 1 & 2 & 3 & 4 & 5 & 6 & 7 & 8 & 9 \\
\hline 0 & $5 \mathrm{~ms}$ & D & $S$ & U & $\mathrm{U}$ & $\mathrm{U}$ & D & $\mathrm{S}$ & $\mathrm{U}$ & $\mathrm{U}$ & $\mathrm{U}$ \\
\hline 1 & $5 \mathrm{~ms}$ & $\mathrm{D}$ & $\mathrm{S}$ & $\mathrm{U}$ & $\mathrm{U}$ & $\mathrm{D}$ & $\mathrm{D}$ & $\mathrm{S}$ & $\mathrm{U}$ & $\mathrm{U}$ & $\mathrm{D}$ \\
\hline 2 & $5 \mathrm{~ms}$ & $\mathrm{D}$ & S & $\mathrm{U}$ & $\mathrm{D}$ & $\mathrm{D}$ & $\mathrm{D}$ & $S$ & $\mathrm{U}$ & $\mathrm{D}$ & $\mathrm{D}$ \\
\hline 3 & $10 \mathrm{~ms}$ & $\mathrm{D}$ & S & $\mathrm{U}$ & $\mathrm{U}$ & $\mathrm{U}$ & $\mathrm{D}$ & $\mathrm{D}$ & $\mathrm{D}$ & $\mathrm{D}$ & $\mathrm{D}$ \\
\hline 4 & $10 \mathrm{~ms}$ & $\mathrm{D}$ & S & $\mathrm{U}$ & $\mathrm{U}$ & $\mathrm{D}$ & $\mathrm{D}$ & $\mathrm{D}$ & $\mathrm{D}$ & $\mathrm{D}$ & $\mathrm{D}$ \\
\hline 5 & $10 \mathrm{~ms}$ & $\mathrm{D}$ & S & $\mathrm{U}$ & $\mathrm{D}$ & $\mathrm{D}$ & $\mathrm{D}$ & $\mathrm{D}$ & $\mathrm{D}$ & $\mathrm{D}$ & $\mathrm{D}$ \\
\hline 6 & $5 \mathrm{~ms}$ & D & $S$ & $\mathrm{U}$ & $\mathrm{U}$ & $\mathrm{U}$ & D & $S$ & $\mathrm{U}$ & $\mathrm{U}$ & $\mathrm{D}$ \\
\hline
\end{tabular}




\section{DOWNLINK SYNCHRONIZATION PRINCIPLE ANALYSIS}

To achieve downlink synchronization in the TD-LTE system design, the following signals are transmitted in the downlink direction: the Primary Synchronization Signal(PSS) and the Secondary Synchronization Signal(SSS).

\section{A. Generation and Mapping of Primary Synchronization Signals}

The generation of the primary synchronization signal sequence is relatively simple, which is obtained by the Zadoff-Chu sequence in the frequency domain ${ }^{[2]}$ :

$$
d_{u}(n)=\left\{\begin{array}{cc}
e^{-j \frac{\pi u n(n+1)}{63}} & n=0,1, \ldots, 30 \\
e^{-j \frac{\pi u(n+1)(n+2)}{63}} & n=31,32, \ldots, 61
\end{array}\right.
$$

The index $u$ of the Zadoff-Chu root sequence is given by the following table ,see Table II.

TABLE II. PSS ROOT INDEX

\begin{tabular}{|c|c|}
\hline$N_{\mathrm{ID}}^{(2)}$ & Root index $u$ \\
\hline 0 & 25 \\
\hline 1 & 29 \\
\hline 2 & 34 \\
\hline
\end{tabular}

Different cell ID groups are numbered as $N_{\mathrm{ID}}^{(2)}$, using different primary synchronization sequences.

Physical resource mapping: The primary synchronization signal sequence is mapped to 62 subcarriers in the frequency domain near the DC subcarriers; in the time domain, it is mapped to the 3rd OFDM symbol of the 1st and 6th subframes. Note that five subcarriers are reserved on both sides of the primary synchronization signal mapping location, and no data symbols are mapped.

\section{B. Generation and Mapping of Secondary Synchronization Signals}

The secondary synchronization signal is a cascade sequence consisting of two pseudo-random sequences of length 31 . Wherein the sequence of subframe 0 and subframe 5 is not the same and is defined as follows:

$$
\begin{aligned}
& d(2 n)= \begin{cases}s_{0}^{\left(m_{0}\right)}(n) c_{0}(n) & \text { in subframe } 0 \\
s_{1}^{\left(m_{1}\right)}(n) c_{0}(n) & \text { in subframe } 5\end{cases} \\
& d(2 n+1)= \begin{cases}s_{1}^{\left(m_{1}\right)}(n) c_{1}(n) z_{1}^{\left(m_{0}\right)}(n) & \text { in subframe } 0 \\
s_{0}^{\left(m_{0}\right)}(n) c_{1}(n) z_{1}^{\left(m_{1}\right)}(n) & \text { in subframe } 5\end{cases}
\end{aligned}
$$

where $0 \leq n \leq 30$. The indices $m_{0}$ and $m_{1}$ are derived from the physical layer cell group $N_{\mathbb{I D}}^{(1)}$ according to

$$
\begin{aligned}
& m_{0}=m^{\prime} \bmod 31 \\
& m_{1}=\left(m_{0}+\left\lfloor m^{\prime} / 31\right\rfloor+1\right) \bmod 31 \\
& m^{\prime}=N_{\mathrm{ID}}^{(1)}+q(q+1) / 2, \quad q=\left\lfloor\frac{N_{\mathrm{ID}}^{(1)}+q^{\prime}\left(q^{\prime}+1\right) / 2}{30}\right\rfloor, \quad q^{\prime}=\left\lfloor N_{\mathrm{ID}}^{(1)} / 30\right\rfloor
\end{aligned}
$$

The two sequences $s_{0}^{\left(m_{0}\right)}(n)$ and $s_{1}^{\left(m_{1}\right)}(n)$ are defined as two different cyclic shifts of the m-sequence $\tilde{s}(n)$ according to

$$
\begin{aligned}
& s_{0}^{\left(m_{0}\right)}(n)=\tilde{s}\left(\left(n+m_{0}\right) \bmod 31\right) \\
& s_{1}^{\left(m_{1}\right)}(n)=\tilde{s}\left(\left(n+m_{1}\right) \bmod 31\right)
\end{aligned}
$$

where $\tilde{s}(i)=1-2 x(i), 0 \leq i \leq 30$, is defined by

$$
x(\bar{i}+5)=(x(\bar{i}+2)+x(\bar{i})) \bmod 2, \quad 0 \leq \bar{i} \leq 25
$$

With initial conditions

$$
x(0)=0, \quad x(1)=0, \quad x(2)=0, \quad x(3)=0, \quad x(4)=1 .
$$

The two scrambling sequences $c_{0}(n)$ and $c_{1}(n)$ depend on the primary synchronization signal and are defined by two different cyclic shifts of the m-sequence $\tilde{c}(n)$ according to

$$
\begin{aligned}
& c_{0}(n)=\tilde{c}\left(\left(n+N_{\mathrm{ID}}^{(2)}\right) \bmod 31\right) \\
& c_{1}(n)=\tilde{c}\left(\left(n+N_{\mathrm{ID}}^{(2)}+3\right) \bmod 31\right)
\end{aligned}
$$

where $N_{\mathrm{ID}}^{(2)} \in\{0,1,2\}$ is the physical-layer identity within the physical-layer cell identity group $N_{\mathrm{ID}}^{(1)}$ and $\tilde{c}(i)=1-2 x(i), 0 \leq i \leq 30$, is defined by:

$$
x(\bar{i}+5)=(x(\bar{i}+3)+x(\bar{i})) \bmod 2, \quad 0 \leq \bar{i} \leq 25
$$

With initial conditions

$$
x(0)=0, \quad x(1)=0, \quad x(2)=0, \quad x(3)=0, \quad x(4)=1 \text {. }
$$

The scrambling sequences $z_{1}^{\left(m_{0}\right)}(n)$ and $z_{1}^{\left(m_{1}\right)}(n)$ are defined by a cyclic shift of the m-sequence $\tilde{z}(n)$ according to $z_{1}^{\left(m_{0}\right)}(n)=\tilde{z}\left(\left(n+\left(m_{0} \bmod 8\right)\right) \bmod 31\right)$

$$
z_{1}^{\left(m_{1}\right)}(n)=\tilde{z}\left(\left(n+\left(m_{1} \bmod 8\right)\right) \bmod 31\right)
$$

where $\tilde{z}(i)=1-2 x(i), 0 \leq i \leq 30$, is defined by

$$
x(\bar{i}+5)=(x(\bar{i}+4)+x(\bar{i}+2)+x(\bar{i}+1)+x(\bar{i})) \bmod 2, \quad 0 \leq \bar{i} \leq 25
$$

With initial conditions

$$
x(0)=0, \quad x(1)=0, \quad x(2)=0, \quad x(3)=0, \quad x(4)=1 .
$$

Physical resource mapping ${ }^{[3]}$ : The secondary synchronization signal is mapped to 62 subcarriers in the frequency domain near the DC subcarrier; in the time 
domain, it is mapped to the last OFDM symbol of subframe 0 and 5 . Note that the secondary synchronization sequences mapped to subframe 0 and 5 are not the same. Similar to the primary synchronization signal, the five subcarriers are reserved on both sides of the secondary synchronization signal mapping location, and no data symbols are mapped anymore.

\section{TIME FREQUENCY SYNCHRONIZATION ALGORITHM}

Through the detection of PSS and SSS to obtain time and frequency synchronization and detection of cell identification number (Cell ID), time-frequency synchronization acquired. There are 504 Cell IDs, divided into 168 groups of 3 . $N_{\mathrm{ID}}^{\text {cell }}=3 N_{\mathrm{ID}}^{(1)}+N_{\mathrm{ID}}^{(2)}$, where $N_{\mathrm{ID}}^{\text {cell }}$ is the Cell $\mathrm{ID}, N_{\mathrm{ID}}^{(1)}$ is the packet number, which are 168 values, from 0 to 167. $N_{\mathrm{ID}}^{(2)}=($ Cell ID $) \bmod 3$ represents the number in the group, and there are three values, from 0 to 2 .

The following figure depicts the entire synchronization process, including the following steps, see Fig. 3.

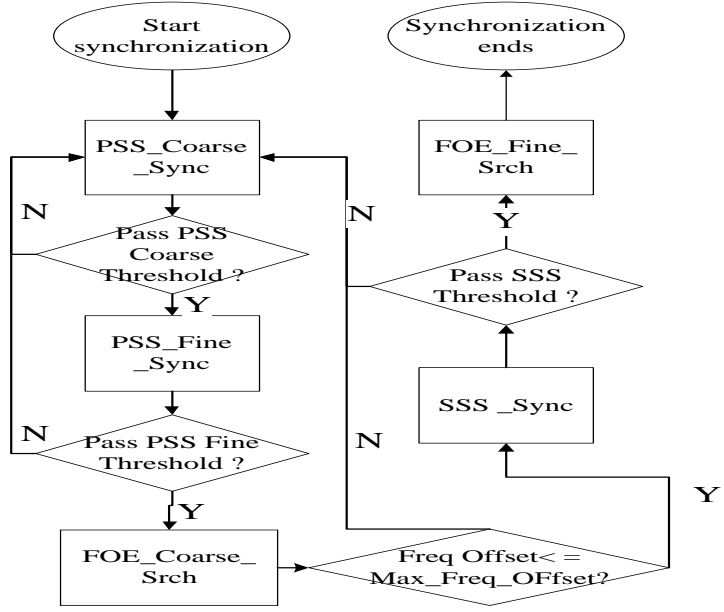

Figure 3. Time-frequency synchronization process

(1) PSS coarse synchronization and PSS fine synchronization;

(2) coarse frequency offset estimation;

(3) SSS synchronization;

(4) fine frequency offset estimation.

A. PSS Synchronization

PSS synchronization is shown as below, see Fig. 4.

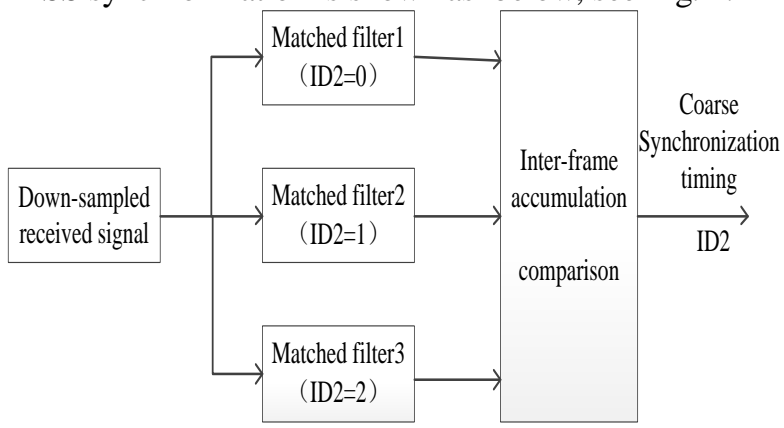

Figure 4. PSS coarse synchronization block diagram
The signal is received using three low-pass matched filters with local PSS signals corresponding to different ID2. In order to reduce the computational complexity, it is possible to first down-sample, to correlate at a coarse precision(coarse synchronization), and then to open a window of the right size around the position to do it, and to correlate it with finer precision(fine synchronization).

When coarse synchronization is performed, the received time-domain signal is down-sampled by 16 times, and then the corresponding process is performed in parallel using the matched filter of the three kinds of PSS, and the inter-frame accumulation is performed to find the peak of the correlation operation. Since the PSS of the previous and subsequent frames is the same, the PSS coarse synchronization finishes the half-frame boundary timing of 16 sample points.

The number of sampling points of one OFDM symbol of the received signal is $N_{s y m}, 16$ times down sampling, $N=N_{s y n} / 16, r(k)$ is the down-sampled received signal, and $s_{I D 2}(k)$ is the local PSS signal.

$$
\begin{aligned}
& c(n)=\sum_{l=0}^{N-1} r(n+l) s_{I D 2}^{*}(l) \\
& p(n)=\sum_{l=0}^{N-1}|r(n+l)|^{2}
\end{aligned}
$$

The normalized correlation values are as follows:

$$
M(n)=\frac{|c(n)|^{2}}{(p(n))^{2}}
$$

Where $\mathrm{n}$ is from 0 to $N w-1, N w$ is the size of the observation window, the number of samples after downsampling is the window size . In order to improve performance, we do the inter-frame accumulation, if the accumulated $N_{1}$ frame, then

$$
M(n)=\sum_{i=1}^{N_{1}-1} M\left(n+i \times N_{w}\right)
$$

If $M(n)$ is less than the threshold of coarse synchronization, re-do coarse synchronization.

The coarse synchronization result can be expressed as:

$$
\begin{gathered}
\text { position }_{\text {coarse }}=\arg \max _{n}|M(n)| \\
I D 2=\arg \max _{I D 2}|M(n)|
\end{gathered}
$$

In the PSS fine synchronization process, the position $_{\text {coarse }}$ 
can be known from the coarse synchronization result of the PSS synchronization, and then a small window is opened near the position $_{\text {caarse }}$, and the fine synchronization can be completed by the correlation calculation.

\section{B. SSS Synchronization}

The position of PSS and $N_{\mathrm{ID}}^{(2)}$ has been obtained by PSS synchronization. From the fixed position relationship between PSS and SSS, SSS can be found, and FFT transform to the frequency domain to extract the data on the middle 62 sub-carriers. Then $168 \times 2$ kinds of SSS with the sequence length of 62 and the correlation signals are carried out in the frequency domain. Since the SSS of the first and second frame of a radio frame is different, the frame boundary can be determined based on the half frame boundary determined by the aforementioned PSS.

The traditional secondary synchronization signal synchronization algorithm is a full search correlation detection algorithm, which needs to traverse the 168 kinds of possible secondary synchronization signal sequence. Since the secondary synchronization sequences of the first and second frames are not the same, the secondary synchronization algorithm requires $168 \times 2$ correlation calculations with length 62 sequences, and the computational complexity is very large. Therefore, in order to reduce the computational complexity, we use the odd-sequence and even-sequence separation detection methods to design and simplify the secondary synchronization signal detection algorithm, which can greatly reduce the complexity of SSS synchronization.

Assuming $r(k)$ is the received SSS sequence, $d_{0}(i, k)$ is the SSS reference signal on the 0th subframe corresponding to the $\mathrm{i}$-th cell group $\mathrm{ID}, d_{5}(i, k)$ is the 5 th sub-frame corresponding to the $\mathrm{i}$-th cell group ID Of the SSS reference signal. The secondary synchronization algorithm is described as below:

$$
\begin{aligned}
& \begin{cases}\operatorname{cov} 0(i)=\sum_{k=1}^{31} r(k) \times d_{0}(i, k) & 0 \leq i \leq 30 \\
\operatorname{cov} 5(i)=\sum_{k=1}^{31} r(k) \times d_{5}(i, k) & 0 \leq i \leq 30\end{cases} \\
& {[\max 0, \text { index } 0]=\max (a b s(\operatorname{cov} 0))}
\end{aligned}
$$

Its concrete realization is shown as below, see Fig. 5.

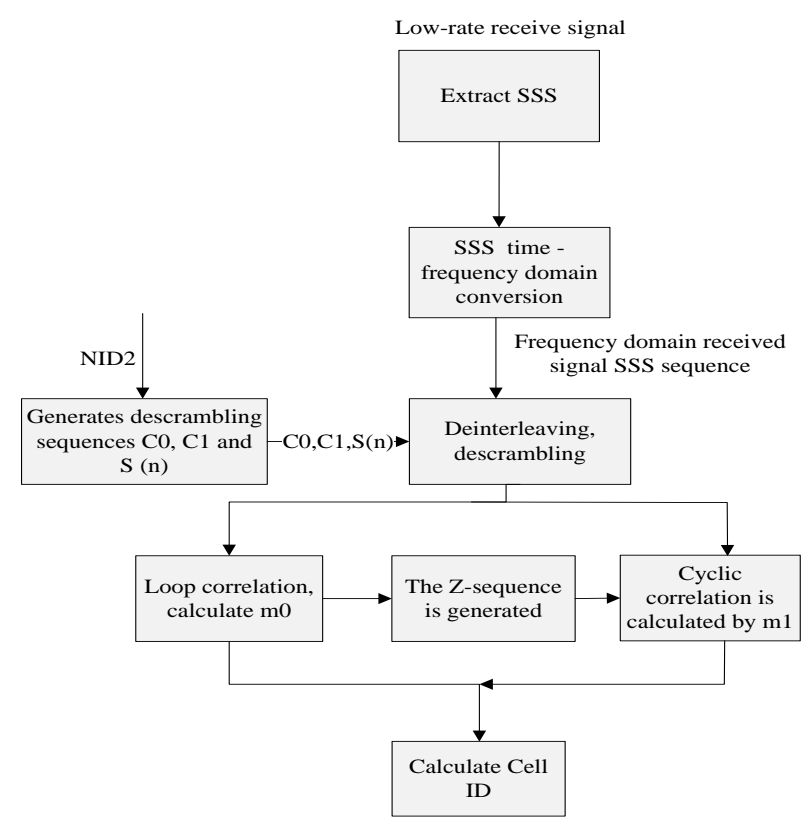

Figure 5. Secondary synchronization process

The method of parity-division separation of SSS saves 18910 times of cross-correlation calculation compared with the traditional method of $168 \times 2 \times 62$ operations. The number of computing times is $1 / 10$ of the traditional correlation detection method. At the same time, the traditional correlation detection algorithm needs more complex accumulator and multiplier, where the hardware resource requirements are higher.

\section{TIME-FREQUENCY SYNCHRONIZATION MODULE ALGORITHM SIMULATION}

\section{A. Cell ID Detection}

The following figure shows AWGN channel, timefrequency synchronization process, 2-frame accumulation, cell ID of the error detection probability curve, the simulation of 5000 times, see Fig. 6.

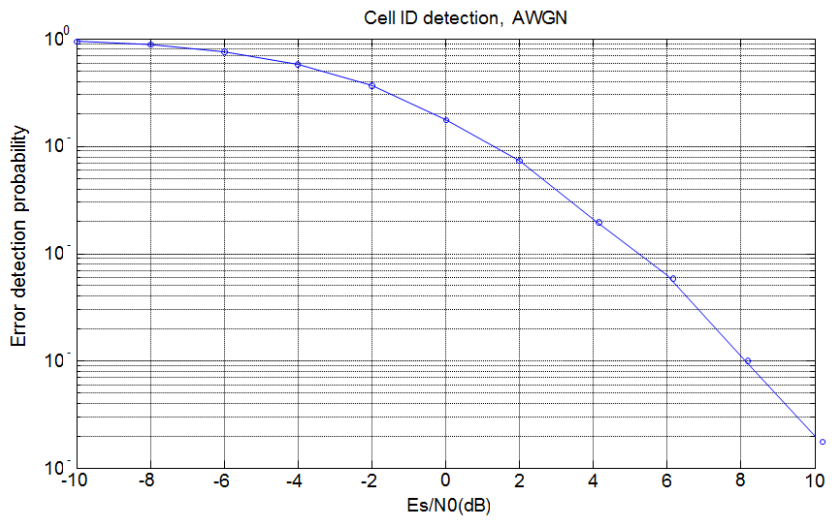

Figure 6. Time-frequency synchronization algorithm cell ID error detection probability curve 


\section{B. The Effect of Frequency Offset on Timing} Synchronization Correctness

Time-frequency synchronization algorithm has accomplished the cell group ID acquisition, time synchronization and frequency estimation functions. Synchronization performance under AWGN channel conditions, fixed frequency offset: $0 \mathrm{~Hz}, 2.5 \mathrm{kHz}, 5 \mathrm{kHz}$, $7.5 \mathrm{kHz}$ and $10 \mathrm{kHz}$, the timing synchronization performance is shown as below, see Fig. 7 .

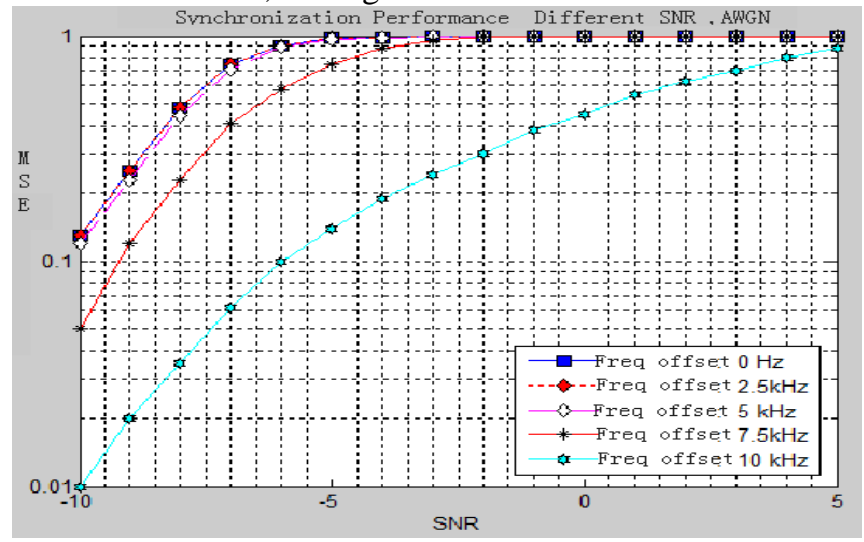

Figure 7. The Effect of Frequency Offset on Timing Synchronization Correctness

It can be seen from the above figure that the frequency offset in the range of $5 \mathrm{kHz}$ has little effect on the timing synchronization performance, and the error rate of timing synchronization greater than $7.5 \mathrm{kHz}$ will become larger when AWGN channel conditions are used.

\section{CONCLUSION}

LTE network is becoming more and more mature, which brings great convenience to mobile internet service in people's life. LTE network coverage requirements are becoming higher, especially in indoor senarios. This paper discusses a new multi-band multi-mode wireless network coverage system - the active optical distribution system, and analyzes the advantages and application characteristics, focusing on TD-LTE primary synchronization signal and secondary synchronization signal performance analysis. Time and frequency synchronization algorithm is simulated, and the simulation results show that a range frequency offset has little effect on timing synchronization accuracy. In the TD-LTE secondary synchronization signal process, the amount of computation is huge, the system resource requirements are high. The parity separation detection method in this paper provides 18910 times less computational complexity than the traditional full-search method. The computational complexity is only one-tenth of the traditional correlation detection method, which reduces the computational burden and reduces the hardware cost.

\section{REFERENCES}

[1] 3GPP TS 36.211 V8.6.0. Evolved Universal Terrestrial Radio Access (E-UTRA)Physical Channels and Modulation (Release 8).March 2009.

[2] 3GPP TS 36.212 V8.2.0. Evolved Universal Terrestrial Radio Access (E-UTRA)Multiplexing and Channel Coding. March 2008.

[3] 3GPP TR36.913 V8.0.0. Requirements for further advancements for E-UTRA (LTE-Advanced). June 2008. 